\title{
Los desafíos a la reunificación de China y Taiwán: la Ley Antisecesión (2005) y el Acuerdo Marco de Cooperación Económica (2010)
}

\author{
The challenges for the reunification between China and \\ Taiwan: the Anti-Secession Law (2005) and the Economic \\ Cooperation Framework Agreement (2010)
}

ISABEL RODRÍGUEZ ARANDA*

Rev. Bras. Polít. Int. 54 (1): 105-124 [2011]

\section{Introducción}

El presente artículo tiene por objetivo analizar el problema de la reunificación entre la República Popular China y Taiwán en la última década, en el marco de dos hitos: por un lado, la promulgación de la Ley Antisecesión del 14 de marzo de 2005 y, por otro, la firma del Acuerdo Marco de Cooperación Económica el 30 de junio de 2010. Este problema de la reunificación, que se inicia desde la huida del gobierno nacionalista chino a la isla en 1949, se mantiene vigente hasta la actualidad como un conflicto sin solución y en statu quo por tres razones que consideramos en este análisis: en primer lugar, por la intransable no renuncia de parte de China continental a recuperar la soberanía sobre Taiwán, y con ello, la constante amenaza del uso de la fuerza militar; en segundo lugar, por el proceso taiwanés de construcción de soberanía interna, westfaliana e interdependiente; y, en tercer lugar, la interdependencia económica entre Taiwán y China. ${ }^{1}$

En este contexto, analizaremos el escenario actual del problema de la reunificación desde dos perspectivas teóricas de la disciplina de las Relaciones Internacionales. Primero, desde el realismo, donde la Ley Antisecesión muestra los códigos de relevancia de los temas de la política tradicional de los Estados: territorio y soberanía. Y segundo, desde el liberalismo, específicamente, desde la perspectiva de la interdependencia compleja de las relaciones económicas que impulsan dinámicas de cooperación e integración entre los Estados, siendo el mejor ejemplo la firma del acuerdo económico de 2010. Asimismo, ambos enfoques se aplicarán al

\footnotetext{
* Profesora en el Departamento de Ciencia Política y Relaciones Internacionales de la Universidad Alberto Hurtado, Chile (irodrigu@uahurtado.cl).

1 Este artículo es parte del Proyecto de Investigación N. 11100096 financiado por el Fondo Nacional de Desarrollo Científico y Tecnológico (FONDECYT) de Chile.
} 
concepto de soberanía utilizando el planteamiento de Stephen Krasner (2001) que diferencia distintos tipos de soberanía, como son: la legal internacional, la interna, la westfaliana y la interdependiente, con el propósito de comprender la existencia y funcionamiento de Taiwán como actor internacional entendiéndolo como un Estado de facto pero carente de soberanía legal internacional. De este modo, nuestro propósito es evaluar el nuevo escenario de las relaciones sino-taiwanesas y los desafíos que se presentan para avanzar hacia una reunificación, o bien, para avanzar hacia la independencia de la isla en un escenario post-acuerdo comercial.

En Relaciones Internacionales, el liberalismo parte de una visión positiva de la naturaleza humana y, por lo tanto, de la convicción que las relaciones entre Estados pueden ser cooperativas más que conflictivas. Dentro de este marco analítico, un eje de análisis que promueve la cooperación económica como dinámica favorable para la ausencia de conflicto es el liberalismo de la interdependencia, el cual enfatiza los lazos económicos a través del intercambio mutuo. Según este enfoque, cuando todos los actores, gobiernos y personas, se centran en el proceso de modernización, crecimiento económico y desarrollo, surgen bases favorables para la cooperación a largo plazo que pueden ayudar a disminuir las posibilidades de conflictos en otras áreas como puede ser la política y militar. Para explicar esta realidad, Keohane y Nye (1993) proponen el concepto de interdependencia compleja, que comprende tres características: primero, la existencia de relaciones entre Estados que ya no son solo relaciones entre los líderes de los Estados sino que hay relaciones entre distintos actores como son las transgubernamentales y transnacionales; segundo, hay un espacio propicio para las relaciones transnacionales fuera de los canales estatales, aquí por ejemplo, las relaciones entre empresarios reforzarían e incrementarían las relaciones comerciales; y tercero, las relaciones internacionales toman características de la política doméstica donde la fuerza militar ya no es lo más importante y sí lo son las habilidades de negociación y los temas no políticos.

En resumen, la modernización incrementa los niveles de interdependencia entre los Estados y promueve relaciones internacionales más cooperativas que se formalizan en acuerdos bilaterales o multilaterales garantizando reciprocidad y permanencia en el tiempo. Por lo tanto, estos acuerdos, son normas que regulan la conducta de los actores, predicen conductas y construyen una percepción positiva del otro y con ello la confianza mutua de las partes.

Por otra parte, el enfoque realista considera que los Estados defienden su interés nacional dentro de un sistema internacional anárquico con el objetivo de adquirir y ejercer poder (WALTZ, 1959, 160). En consecuencia, son los Estados las unidades primarias del sistema internacional que formulan estrategias y desarrollan capacidades para defender sus intereses y lograr la predictibilidad del actuar de los otros Estados (JACKSON \& SORENSEN, 2003, 75). Es decir, los Estados actúan racional y defensivamente en función de su supervivencia, cuyo elemento fundamental es la preservación de la soberanía, para lo cual existe la posibilidad de formar alianzas, pero de carácter defensivo de acuerdo a esos intereses. 
Por consiguiente, para el problema de la reunificación de China y Taiwán es posible encontrar estas dos tendencias claramente identificables. Una tendencia hacia la permanencia de principios realistas en la toma de decisiones y demandas por territorio y soberanía, siendo parte de esto también la política de alianzas como es el caso de Taiwán con un actor extrarregional, EE. UU. y, una segunda tendencia a la interdependencia económica, donde las relaciones económicas, principalmente los flujos de inversión, generan un proceso de regionalización que activa la cooperación y hace cada vez más costoso activar el conflicto político, proceso impulsado por la modernización económica llevada a cabo por la R.P. China en las últimas tres décadas. Ambos aspectos proyectados en un punto de tope, a saber, la indefinición de la soberanía de Taiwán.

Centraremos el análisis en cuatro aspectos que estructuran la división del desarrollo del artículo. Primero, revisaremos los antecedentes del proceso de separación de China y Taiwán y los principales hitos que han marcado la hasta ahora categórica no solución del conflicto; en segundo lugar, analizaremos la Ley Antisecesión del año 2005 y el rol de EE. UU. en el conflicto como factor de no solución; en tercer lugar, la importancia de la firma del Acuerdo Marco de Cooperación Económica en 2010 como punto que activa el diálogo entre las partes; $y$, en cuarto lugar, los factores políticos internos en Taiwán que ayudan al statu quo del conflicto pero que permiten reconocer la existencia de un Estado de facto que es parte de la comunidad internacional.

El análisis de estos cuatro aspectos nos permitirá sostener y concluir que las variables que han mantenido el conflicto en statu quo hasta ahora, se mantienen constantes a pesar de la promulgación de la Ley Antisecesión, y a su vez se suma una nueva variable que es la interdependencia económica, proceso que se ha formalizado en un tratado de libre de comercio. A partir de este nuevo hito ocurrido el año 2010, se concluye que no hay variables relevantes que permitan proyectar un conflicto armado entre China y Taiwán. Asimismo, el rol antes decisivo de EE. UU. en el conflicto ahora se neutraliza luego de la firma de este acuerdo formal de cooperación económica. Por lo que a partir de estos eventos, dos escenarios son posibles: avanzar hacia una reunificación, o bien, avanzar hacia la independencia de la isla.

\section{Antecedentes del conflicto entre China y Taiwán}

El problema de la reunificación entre China y Taiwán se gesta como consecuencia de la guerra civil interna del país entre nacionalistas y comunistas. Desde 1911 gobernaba en China continental el Kuomintang, que fundó la República de China, periodo que se extiende hasta 1949, cuando Mao Zedong, luego de la victoria comunista, funda la República Popular China (R.P. China). Como consecuencia de esto, el gobierno nacionalista del Kuomintang huye a la isla de Taiwán sosteniendo ser el legítimo gobierno representante de China, situación 
que fue legitimada por la comunidad internacional al mantener la representación de la República de China en la ONU hasta 1971 (BAILEY, 2002, 115).

El contexto internacional estructurado por la bipolaridad del periodo de guerra fría mantuvo el conflicto «congelado», siendo el apoyo de EE. UU. a Taiwán un factor determinante en el statu quo, pero también un factor que sostiene la no solución del problema hasta nuestros días. Recordemos que este apoyo a Taiwán era parte de la estrategia de EE. UU. en Asia oriental para detener el avance del comunismo, de ahí el apoyo para la permanencia de República de China en la ONU.

No obstante, en 1971 la R.P. China reemplazará a la República de China en la ONU imponiéndose como el legítimo gobierno representante de China. De este modo, Taiwán fue expulsado de la institución y se inició con ello un reconocimiento de los distintos países de la comunidad internacional de la existencia de «Una China» que sería la R.P. China, mientras que Taiwán fue considerado una "provincia rebelde», teniendo que participar en la comunidad internacional bajo el nombre de «Taipei Chino».

En este contexto, en las últimas seis décadas la «cuestión de Taiwán» ha sido una constante dificultad en la política exterior de la R.P. China, principalmente ha constituido un obstáculo en el desarrollo de relaciones diplomáticas y de confianza con EE. UU., que se retomaron con la visita de Richard Nixon a China recién en 1972. Además, ha significado para China una constante lucha diplomática por evitar las relaciones diplomáticas formales de Taiwán con otros países. Todavía quedan países que tienen relaciones diplomáticas con Taiwán y no con China, principalmente en Centroamérica y África ${ }^{2}$, proyectando esta guerra diplomática hasta el presente. Por ejemplo, en los países que reconocen a la R.P. China, Taiwán tiene la denominada "Oficina Económica y Cultural de Taiwán», que funcionan como embajadas y les permite estar en un constante lobby por el reconocimiento de su condición de Estado autónomo de la China continental.

El conflicto cambia en la década de 1990, matizándose por las percepciones de las partes involucradas, principalmente por parte de Taiwán, que deja de reclamar ser el gobierno representante de la República de China y abre un contexto favorable para que China proponga una formula pacífica para la reunificación y, de alguna manera, pueda frenar la posibilidad de que Taiwán se declare un Estado independiente (LEE, 1995, 55).

Recordemos que en la década de los noventa, se consolida la apertura económica de China continental y con ello su integración a la comunidad internacional, lo que trae como consecuencia un cambio de actitud en la percepción del gobierno comunista sobre la vía que debería tomar el proceso de reunificación con Taiwán, adoptándose una actitud negociadora que se reflejó en una propuesta

2 Entre los países que mantienen relaciones diplomáticas con Taiwán en Centroamérica y el Caribe están: Panamá, Belice, República Dominica, El Salvador, Guatemala, Honduras, Nicaragua, Haití, San Cristóbal y Nieves, Santa Lucía, San Vicente y las Granadinas. Mientras que en África están: Gambia, Santo Tomé y Príncipe, Suazilandia, y Burkina Faso. 
de reunificación pacífica llamada «Un país, dos sistemas» dada a conocer en 1992 por el Presidente de la R.P. China, Deng Xiaoping. Esta propuesta se realizó tomando como referencia la organización que se proponía para los procesos de recuperación de Macao en 1999 y de Hong Kong en 1997. Sin embargo, para el caso de Taiwán agregaba concesiones adicionales, señal de reconocimiento de la complejidad asociada al proceso de división territorial impulsado por un proceso político interno y no producto de la colonización de potencias extranjeras, como fue el caso de Macao por Portugal y Hong Kong por Gran Bretaña (LEE, 1995, 62).

La propuesta señalaba que Taiwán, al volver a la soberanía de la R.P. China, podría mantener intacto su sistema político, su estructura socioeconómica, sus relaciones «no gubernamentales» con terceros países, además de contar con todas las garantías para la plena independencia judicial y legislativa, así como también militar. Es decir, no se enviarían representantes políticos ni militares a la isla, por el contrario, se pondrían a disposición de las autoridades taiwanesas un cierto número de puestos al máximo nivel del gobierno continental (REPÚBLICA POPULAR CHINA, 2000).

No obstante, esta propuesta pacífica de la R.P. China va a coincidir con la etapa inicial del proceso de democratización del sistema político de Taiwán, donde los partidos políticos compiten por las elecciones parlamentarias y luego presidenciales, lo que llevará a hacer uso del tema de la independencia de la isla como parte de sus campañas políticas. Así, el partido opositor en Taiwán, el Partido Progresista Democrático (PPD), tomó como proclama en su campaña la independencia de Taiwán y transformó el tema en una crítica al candidato que representaba la continuidad del gobierno del Kuomintang (KMT), quienes no compartían esta idea de declarar la independencia. Esto provocó una reacción inmediata por parte de la R.P. China, efectuando maniobras militares en el Estrecho de Taiwán que incluyeron el lanzamiento de misiles en las proximidades de las costas taiwanesas (HALBEISEN, 2003, 11). Este episodio de 1996, se conoce como el «conflicto del Estrecho de Taiwán».

El resultado de las elecciones presidenciales dieron la victoria al candidato del hasta ese momento partido gobernante por más de cuarenta años, el KMT, con Lee Teng Hui, reduciendo la tensión entre ambas partes del estrecho e incluso impulsó una iniciativa conjunta de crear la Fundación para el Intercambio en el Estrecho, que consistía en una organización constituida por representantes no gubernamentales de los gobiernos de ambas partes para formalizar contactos y un diálogo que permitiera una solución pacífica al conflicto (RIGGER, 1999, 149).

Sin embargo, en 1999 otro acontecimiento va a tensionar las relaciones en el estrecho, ya que el presidente Lee Teng Hui declararía en un canal de televisión alemán mantener, desde 1991, una relación de Estado a Estado con la R.P. China (HALBEISEN, 2003, 12). Esto provocó la suspensión inmediata de todos los contactos logrados y reforzó la presencia y actividad militar de China en las aguas del estrecho; esta vez, sin embargo, no hubo lanzamiento de misiles. 
Un tercer acontecimiento que llegó a posturas prebélicas fue la víspera de la elección presidencial de Taiwán del año 2000. La creciente democratización de la isla amenazaba, por primera vez en cincuenta años, la supervivencia del poder político del KMT. En efecto, el candidato opositor, Chen Shui-bian, del PPD, demostraba altas posibilidades de ser elegido y una de sus propuestas era su discurso pro independentista. No obstante, al ser elegido Chen Shui-bian como presidente de Taiwán, moderó su discurso y mantuvo el statu quo de la situación de Taiwán respecto a la posible reunificación con China. Además, para su reelección del 2004 su discurso se matizó bastante, tendiendo hacia la moderación y no hacia la confrontación con China, evitando prometer al electorado declarar la independencia de la isla al ser reelecto (HALBEISEN, 2003, 14).

En esta última reelección, la R.P. China estuvo atenta a los acontecimientos de la isla y al discurso de Taiwán y no demoró en recalcar su postura intransigente respecto a un ataque militar en caso de ser declarada la independencia. Así manifestaba que China continental no puede transar la premisa de que Taiwán es una provincia de China, aspecto que se formaliza de forma definitiva con la Ley Antisecesión de 2005. No obstante, el panorama en las últimas elecciones presidenciales de Taiwán provocará cambios importantes en las relaciones con China. En efecto, el triunfo de Ma Yuig Jeou del partido KMT en marzo de 2008, junto al triunfo del mismo partido en las elecciones legislativas de enero del mismo año, son un antecedente de cambio en la perspectiva de relacionamiento con China, a favor del entendimiento y del diálogo, aspecto que se formaliza en la firma del Acuerdo marco de Cooperación Económica de 2010.

\section{La Ley Antisecesión: un hito en los aspectos realistas de la reunificación}

El 14 de marzo de 2005, la R.P. China adopta, en la III sesión de la X Asamblea Nacional Popular (ANP) la Ley Antisecesión en relación al problema de la reunificación con Taiwán, siendo promulgada oficialmente por una orden presidencial del presidente $\mathrm{Hu}$ Jintao el mismo día de su adopción. La votación de la ANP fue unánime en la adopción de la ley y tuvo un apoyo generalizado en la población china.

Para el gobierno chino, esta Ley Antisecesión tenía por objetivo defender la soberanía nacional e integridad territorial de la R.P. China y oponerse a las fuerzas secesionistas que buscan la independencia de Taiwán. Por consiguiente, esta ley, según la perspectiva de la China continental, buscaba mantener la paz y la estabilidad en la región del estrecho de Taiwán y salvaguardar los intereses fundamentales de la nación china. Sin embargo, sus repercusiones fueron bastante preocupantes en cuanto al temor expresado por países de la región, y por EE. UU. en particular, de ser la causa del desencadenamiento armado de un conflicto hasta ahora congelado. Otros países apoyaron a la R.P. China, entre ellos la Unión Europea, por seguir el sentido diplomático de reconocimiento de la existencia de «Una China» pero, principalmente las declaraciones de EE. UU., actor directamente 
involucrado por su tradicional apoyo a Taiwán, alarmaron en su momento sobre las consecuencias de la promulgación de esta ley. Concretamente se temía que con este instrumento legal la R.P. China tuviera mayor legitimidad para el uso de la fuerza en su intento de recuperar la soberanía de la isla.

$\mathrm{Al}$ respecto, si analizamos las declaraciones hacia Taiwán que ha hecho la R.P. China desde 1949, y más todavía desde 1992, fecha en que se propone la creación de «Un país, dos sistemas», podemos observar que la China continental está de acuerdo con una reunificación pacífica con Taiwán. Sin embargo, junto a esta propuesta de reunificación pacífica, China expresa su no renuncia al uso de la fuerza en caso de ser declarada por Taiwán su condición de Estado independiente.

A nuestro juicio, es esto, y no otra cosa, lo que se plasmó en la Ley Antisecesión, es decir, las declaraciones constantes adoptadas por el PCCH se formalizaron en una ley a través de las instituciones del Estado chino y con la participación del pueblo chino a través de la ANP. La pregunta que surge entonces es si este paso desde las declaraciones de los líderes políticos chinos a la creación de una ley puede desencadenar el conflicto armado. Nuestra respuesta es que dicha ley solo formaliza la postura que China siempre ha tenido al respecto y no quita ni agrega mayores elementos que activen el conflicto con Taiwán, aspecto que queda demostrado al constatar que de 2005 a 2010 no hubo mayores problemas en la relación. En este sentido, Taiwán siempre ha sabido que al declarar su independencia será atacada por la China continental, y esta última, sabe que atacar a Taiwán provoca el involucramiento de EE. UU. en el conflicto. Por consiguiente, la conjugación de ambos aspectos es lo que han mantenido el problema de la reunificación constante, pero sin solución (TOW, 2001).

Los trabajos en el tema, en general, coinciden en que EE. UU. tendría dos opciones en caso de que Taiwán declarara su independencia (LEE, 1995; RIGGER, 1999; HALBEISEN, 2000). Un primer escenario sería intervenir a favor de Taiwán en caso de un ataque armado de parte de la R.P. China a la isla, lo que significaría una guerra armada con la R.P. China sin saber exactamente qué tipo de armas posee China y por ende qué capacidad militar real tiene este país para defender su soberanía sobre la isla. El segundo escenario sería no intervenir en caso de que Taiwán fuera atacada por China, con lo que EE. UU. sufriría una pérdida de credibilidad tanto en la región como en el plano internacional.

Desde esta perspectiva, ninguno de los escenarios es conveniente para EE. UU. En este sentido, este país tiene que hacer todo lo posible para prevenir que Taiwán declare su independencia. Por esto, EE. UU. es claro en reconocer el principio de «Una China» y no apoyar la incorporación de Taiwán a alguna organización internacional intergubernamental. En consecuencia, sin un apoyo seguro de EE. UU., es casi imposible que Taiwán declare su independencia. Esta articulación de actores y roles hace que el problema de la reunificación ChinaTaiwán se mantenga hasta ahora en statu quo, sin variación en la postura de las partes involucradas, y sin una posible solución en el corto y mediano plazo. 
Apoyan estas buenas relaciones entre China y EE. UU. los comunicados conjuntos que han sido la base para mantener buenas relaciones diplomáticas entre ambos países. Dichos comunicados son tres: el de 1972, el de 1979 y el de 1982. En general, estas declaraciones han permitido a las partes dar señales claras de la voluntad para desarrollar buenas relaciones entre ambos e incluyen los aspectos más difíciles de reconciliar. Por eso, estos comunicados conjuntos son de gran importancia hasta hoy en las relaciones entre EE. UU. y China en relación a la situación de Taiwán.

Es más, luego de ser firmada la Ley Antisecesión, EE. UU. declaró que era un peligro para la estabilidad y la paz en la región, en un tono muy crítico respecto al actuar de la R.P. China. Al respecto, la respuesta de la R.P. China fue que EE. UU. estaba violando los principios de los comunicados conjuntos de 1972, 1979 y 1982, infringiendo así las normas básicas que regulan las relaciones internacionales y constituyendo una intervención en los asuntos internos de China (DECLARACIÓN DEL MINISTERIO, 2005). En dichos comunicados, ambos reconocen que el asunto de Taiwán es uno de los mayores impedimentos para desarrollar buenas relaciones, y por eso mismo China es muy clara en incorporar tres cosas: ser el legítimo gobierno de China, que Taiwán es una provincia de China, y que la recuperación de la isla es un asunto interno de China. Por su parte, EE. UU. declara en los comunicados que reconoce la existencia de una China donde Taiwán es parte de ella, comprometiéndose a reducir sus fuerzas e instalaciones militares que posee en Taiwán.

En consecuencia, el rol de EE. UU. en el conflicto es ambiguo y es también factor de statu quo; parece buscar buenas relaciones con China pero a la vez tiene una relación con Taiwán desde el periodo de Guerra Fría que involucra relaciones comerciales, incluyendo la venta de armamento. Sin embargo, un conflicto en el Asia Oriental no es conveniente para EE. UU. por la inestabilidad política y económica que se produciría y porque es un conflicto por temas de soberanía que se aleja de las actuales prioridades de la política exterior norteamericana, como es el terrorismo y su articulación en el Medio Oriente, así como su ramificación a países también del Asia Oriental. En este sentido, China es un aliado en cuanto es un actor válido para el diálogo con Corea del Norte y también es amenazada por la presencia de grupos fundamentalistas islámicos en su región de Xinjiang (RODRÍGUEZ, 2010, 182).

Desde esta perspectiva, el tema de la soberanía sigue siendo un aspecto fundamental para la R.P. China y una base de legitimidad del PCCH en el poder. Para el PCCH, el tema de mantener la integridad territorial de China y recuperar Taiwán ha sido una bandera de lucha constante que une al pueblo por una causa común, por lo que renunciar a esta posibilidad es deslegitimarse ante la población como un gobierno que no defiende los intereses del país (RODRÍGUEZ, 2010, 173). Además, existen otros grupos al interior de China ubicados en zonas geográficas determinadas del país que reclaman autonomía y renunciar a 
la soberanía de Taiwán sería sentar un precedente para alentar a estos grupos a exigir su independencia. De este modo, la desintegración territorial de China y la pérdida de poder del PCCH, llevarían a una inestabilidad política afectando a la modernización económica del país que es otra base de legitimidad del mismo partido comunista ante la población.

Por otra parte, está la postura e influencia de los militares en el sistema político chino, para quienes es inconcebible renunciar a la soberanía sobre Taiwán. De hecho se considera que China es débil militarmente para atacar a Taiwán, quien posee armamento más sofisticado y el apoyo militar de EE. UU. No obstante, el presupuesto militar en China ha estado aumentando con los años, y precisamente en la misma Asamblea Nacional Popular del año 2005 se aprobó un incremento del presupuesto destinado a defensa de un $12,5 \%$, cifra de dos dígitos que mantiene hasta el 2010, aprobándose un incremento del presupuesto en un 7,5\% (PRESUPUESTO DE DEFENSA DE CHINA, 2010). Esto es una variable a considerar sobre cómo la China continental se está preocupando de reforzar un recurso de poder tradicional, como es su capacidad militar, más aún manteniendo conflictos territoriales importantes en la región, como el caso de Taiwán y la demanda de soberanía sobre las Islas Spratly, ubicadas en el mar del sur de China y que disputa con otros países de la región.

Desde estas variables y como conclusión, el tema de la soberanía tanto para China como para Taiwán mantiene una interpretación tradicional, realista en el análisis de las relaciones internacionales, muy alejada de las nuevas variables que la integración económica está produciendo en la región y que ahora se hace palpable en el acuerdo económico firmado entre ambas chinas.

\section{El Acuerdo Marco de Cooperación Económica de 2010: un hito en los aspectos liberales de la reunificación}

Además de las variables políticas que giran en torno al principio de soberanía, están también las variables económicas de interdependencia e integración entre las economías de la China continental y de Taiwán (GALEN \& DORN, 2000).

En efecto, ambas regiones cumplen un rol determinado en el crecimiento económico de las partes y del todo. Por una parte, China es la base de la inversión, del asentamiento industrial, de la mano de obra abundante y barata, Hong Kong en cambio, es el centro financiero de la economía china y de la región, mientras que Taiwán es el impulsor de la inversión llevando dinero y empresarios a China continental, principalmente en la parte sur del continente. Además, a esta complementariedad económica se suma la afinidad cultural que no deja de ser un factor relevante en las buenas relaciones de confianza e interdependencia económica que se gesta en esta triada. Recordemos que hay una tendencia comprobable de que las comunidades chinas de ultramar han canalizado inversión a la China continental. 
Desde la apertura económica los taiwaneses invierten en el continente, siendo el periodo 1979-1995 de donde provienen los mayores volúmenes de inversión extranjera. Por su parte China continental se preocupó de ofrecer un marco regulador especialmente ventajoso creado para atraer inversiones para el procesamiento y ensamblaje de exportaciones. En datos más recientes, tenemos que cada año Taiwán invierte en China más de 3000 millones de dólares y, solo en la región de Shanghai, viven y trabajan medio millón de taiwaneses. A su vez, China es el principal comprador de productos taiwaneses y absorbe la cuarta parte de las exportaciones de la isla, por un valor de 65000 millones de dólares (POCH DE FELIU, 2005).

También debe tenerse presente que existen muchos taiwaneses y hongkoneses trabajando en el continente y que muchos habitantes de China continental residen temporalmente en Hong Kong; el resultado del intercambio de experiencias, a partir del creciente flujo de personas entre estas tres regiones de China, genera relaciones transnacionales que favorecen la construcción de vínculos formales entre los gobiernos. En consecuencia, se está utilizando el proceso de integración económica, forjado a partir del intercambio de comercio e inversiones, para vincular las zonas costeras del continente y la isla de Taiwán, por lo que, si bien no existe una integración política entre la R.P. China y Taiwán, ambos territorios se identifican culturalmente y refuerzan su interdependencia económica en un proceso de regionalización espontánea.

Asimismo, ha sido importante el espacio multilateral, en el plano principalmente económico, que ambos países han compartido. Concretamente el Foro de Cooperación del Asia Pacífico. Al analizar los elementos constitutivos del problema de la reunificación de la República Popular China con Taiwán, encontramos que la interdependencia económica entre ambos ha sido un factor de tope en la posibilidad de una radicalización del conflicto. También la interdependencia compleja jugó un papel importante en la reincorporación de Hong Kong a China pese a las diferencias de ambos sistemas. Al mismo tiempo, se demuestra en la actual política exterior de China continental hacia la región, la cual busca la estabilidad, seguridad y cooperación, dejando de lado intentos de hegemonía, lo que muestra una clara relación entre la interdependencia compleja y el multipolarismo en la región. Desde que China adoptó la política de apertura económica en el año 1978, su economía ha estado creciendo a un ritmo anual cercano al $10 \%$, un crecimiento que ha variado desde un $14 \%$ al iniciarse los años noventa, un $10 \%$ a mediados de esa misma década (WU, 1996, 45), para finalmente estabilizarse en torno al $10 \%$ actual, considerando además el impacto de la crisis mundial actual que ha llevado el promedio de crecimiento de China en lo que va del año 2009 a un $7 \%$ aproximado, pero se proyecta una tendencia futura al alza nuevamente (OCDE MEJORA PROYECCIONES, 2010).

Sin embargo, queda abierta la discusión respecto a la compatibilidad de la regionalización económica entre Hong Kong, Taiwán y la R.P. China que tiene 
cabida en el Foro de Cooperación del Asia Pacífico (APEC) de regionalismo abierto unilateral, pero que difícilmente podrá integrarse a este nuevo regionalismo abierto bilateral y posiblemente regional por el problema del no reconocimiento de Taiwán como Estado.

Es justamente lo anterior lo que cambia completamente con el Acuerdo Marco de Cooperación Económica firmado entre China y Taiwán el 29 de junio de 2010. Esto, en la medida que viene a incluir un aspecto más a la integración económica que es favorable a la reunificación pacífica de las partes. Pero ¿qué explica que Taiwán haya aceptado firmar este acuerdo comercial con China? Al respecto, hay variables de naturaleza económica, de política interna y de política internacional.

En lo económico, Taiwán se ha visto muy afectado por la crisis internacional y necesita una pronta recuperación. En lo concreto, aumentar su PIB, que en la actualidad es de un $4,72 \%$, a uno que esté por encima del $6 \%$, además de crear 260000 nuevos empleos (OFICINAS COMERCIALES, 2010). En este sentido, este acuerdo comercial significa para Taiwán aumentar las exportaciones a China por la reducción de tarifas negociadas y tener beneficios para invertir en el continente.

Ambos son mutuamente los principales socios comerciales considerando que el flujo de bienes taiwaneses a China asciende a 80000 millones de dólares al año, mientras que en sentido contrario suma 30000 millones (REINOSO, 2010). A eso se agrega que China está negociando otros acuerdos comerciales, por ejemplo, con ASEAN que involucra un mercado de más de 500 millones de personas reuniendo a importantes economías que compiten con Taiwán, como son Vietnam, Singapur, Indonesia, Filipinas, Tailandia, Malasia, Brunei, Camboya, Myanmar y Laos. Desde este punto de vista, para Taiwán el acuerdo comercial es una estrategia para asegurar que sus exportaciones no pierdan competitividad frente al resto de los socios comerciales de China. En una perspectiva más amplia, esta estrategia apunta entonces a evitar que Taiwán quede marginado del proceso de integración económica de Asia Oriental.

Debemos considerar como beneficio para Taiwán que se trata de un acuerdo comercial asimétrico, es decir, una de las partes obtiene mayores beneficios que la otra. Al respecto, hasta el doble de las exportaciones taiwanesas tendrán derecho a obtener tarifas más bajas que las exportaciones chinas a la isla. Se reducirán y eliminarán los aranceles a la exportación de 539 categorías de productos taiwaneses y a la importación de 267 categorías chinas. Beijing recortará los aranceles a productos de la isla valorados en 13840 millones de dólares, mientras Taipei lo hará a artículos chinos cifrados en 3000 millones (REINOSO, 2010).

Además, el acuerdo no afecta mayormente a los sectores sensibles de la economía taiwanesa como el sector agrícola, porque el acuerdo no incluye una reducción de tasas para productos de este sector. A su vez, logró negociar a su favor aspectos favorables a las exportaciones de los productos más sensibles de la industria taiwanesa, como los plásticos de PVC y productos de alta tecnología, productos a los que China se resistía. 
En lo político, el Acuerdo Marco de Cooperación Económica es un paso importante no solo para las relaciones de interdependencia económica entre las partes, sino también para mejorar las relaciones políticas que puedan favorecer la búsqueda de una solución al conflicto de soberanía que se disputa. Dicho conflicto de soberanía tiene dos aristas. Por una parte, está el reclamo de China de soberanía sobre la isla, y por otra, está el reclamo taiwanés de ser reconocido como un Estado soberano. Para ambas demandas los actores han utilizado distintas estrategias, tales como la vía diplomática, la amenaza del uso de la fuerza, la configuración de alianzas de seguridad, y la prohibición de vínculos y contactos entre los habitantes de ambos territorios.

En consecuencia, lo único que no ha seguido los códigos realistas en relación a la seguridad y demandas de soberanía han sido las relaciones económicas. Aquí ha existido un proceso de regionalización espontánea que al formalizarse como Acuerdo Comercial tiene repercusiones políticas.

En primer lugar, y en clave política, debemos precisar que este Acuerdo es el equivalente a un tratado de libre comercio entre las partes, pero en este caso no es un tratado firmado por dos Estados, ya que China no reconoce la condición de tal a Taiwán. Es entonces un acuerdo firmado por dos organizaciones no gubernamentales con la aprobación de ambos gobiernos. En concreto, el acuerdo fue firmado por Chiang Pin-king, presidente de la Fundación para los Intercambios a través del Estrecho de Taiwán (SEF), y por Cheng Yun-lin, presidente de la Asociación de Relaciones entre ambos lados del Estrecho de Taiwán (ARATS).

Las condiciones para activar el proceso de diálogo y negociación entre China y Taiwán que tiene como resultado la firma del acuerdo de junio de 2010, tienen su origen en la visita del presidente del KMT, Lien Chan, el 29 de abril de 2005, quien viaja a China a entrevistarse con el Presidente de ese país, Hu Jintao, y con ello, aliviar tensiones acumuladas desde la firma de la Ley Antisecesión por China en marzo de 2005.

Dichas tensiones venían de un proceso de más larga data, específicamente desde el primer acercamiento entre China y Taiwán desde la división de 1949, que fue el 27 de abril de 1993 en Singapur, entre Koo Chen-fu, presidente de la SEF, y Wang Daohan, presidente de ARATS, las mismas organizaciones no gubernamentales que han firmado el acuerdo comercial de 2010. Este encuentro de 1993, fue posible por el establecido "Consenso de 1992», por el cual ambas partes aceptan la existencia de una China pero con interpretaciones distintas. Por una parte, China identifica una unidad bajo la República Popular China y, por otra parte, Taiwán identifica esta unidad bajo la República de China.

Estas diferencias dentro del marco de "consenso" logrado dificultan la construcción de confianzas mutuas, lo que termina anulando el proceso de acercamiento cuando en junio de 1999 el presidente de Taiwán, Lee Teng Hui, viaja a EE. UU. y en sus declaraciones especifica que las relaciones con China son estatales. A esto se suma, que en 2000 es elegido como presidente de Taiwán Chen 
Shui-bian, representante del partido opositor al KMT, el PPD, que detenta una posición sin duda a favor de la independencia a la isla. Este proceso de alejamiento y estancamiento de los contactos, culmina con la firma de la Ley Antisecesión de 2005 por parte de China, con la cual ampara constitucionalmente el uso de la fuerza en caso que la provincia de Taiwán declare su independencia.

En este contexto, el acuerdo comercial de 2010 es el resultado de la activación de un proceso de acercamiento y de un proceso de construcción de confianzas entre las partes. Clave fueron las últimas elecciones presidenciales en Taiwán, donde retorna al poder el partido KMT, en la persona de Ma Ying-jeou, en mayo de 2008. Con ello, se retomó el contacto con China con resultados inmediatos, firmando en el mes de junio un acuerdo para reiniciar los vuelos directos regulares entre ambas partes y en noviembre se firmaron cuatro acuerdos sobre transporte aéreo y marítimo, lazos postales y seguridad marítima. Y, por último, en diciembre de 2009, se firman tres acuerdos más referidos a la cooperación pesquera, inspección, estándares y metrología de productos agrícolas.

La cooperación económica permite un acercamiento de intereses, negociar en un ámbito común, la construcción de confianzas, y cambiar positivamente las percepciones del otro. Bajo esta perspectiva de análisis del enfoque liberal de las relaciones internacionales, el actual acercamiento y negociación sobre diversos temas es beneficioso para las buenas relaciones de China y Taiwán, y para activar un posible proceso de reunificación. En consecuencia, con el Acuerdo comercial se produce un acercamiento económico entre iguales, que puede llevar a un acercamiento político entre iguales en un proceso de reunificación simétrico. $\mathrm{O}$ bien una relación entre iguales que vaya otorgando paulatinamente un reconocimiento a la soberanía legal internacional de Taiwán, en un marco que a la vez impulse un proceso de mayor integración social y política con China.

En este sentido hipotético, el que Taiwán obtenga su independencia no significaría necesariamente un mayor alejamiento del continente y la isla; por el contrario, también puede activar un proceso de integración entre iguales, que afiance un proceso de unidad paulatino que se ha iniciado con la cooperación económica entendida como un tratado de libre comercio, pero que puede seguir hacia espacios de mayor integración, como son una unión aduanera o un mercado común.

Es más, y siguiendo la teoría neofuncionalista (HAAS, 1958) que se enmarca en la perspectiva liberal, la colaboración funcional exitosa en un sector genera la necesidad de colaboración funcional en otros sectores. Es lo que Mitrany (1943) llamaba un proceso de spill-over, es decir, un proceso de ramificación en la colaboración en constante aumento, que podría llegar incluso a la propia soberanía estatal. Es el caso del proceso de formación de la Unión Europea, donde se crea la Comunidad Económica del Carbón y el Acero (CECA) en 1956 y se produce su posterior desbordamiento con la creación de la Comunidad Económica Europea (CEE) y la Comunidad Europea de la Energía Atómica (EURATOM), 
donde la integración alcanza cada vez con mayor complejidad y profundidad los temas políticos. Aquí la soberanía se transfiere a instituciones supranacionales que toman las decisiones de acuerdo a los intereses comunitarios y no de los Estados individuales. Con ello, se comparte soberanía sin quitar soberanía a los Estados miembros de la Unión Europea, proceso que se extiende hasta la firma del Tratado de Lisboa de 2010.

En consecuencia, según los neofuncionalistas, en la construcción de estos procesos de integración se mantiene la consideración de base de entender a las partes involucradas como grupos competidores con intereses opuestos. No obstante, se espera que como parte del proceso de ramificación, las elites políticas resuelvan esas diferencias de intereses porque perciben que la integración política es la mejor vía de satisfacer sus intereses. De alguna manera, la supranacionalidad en la visión neofuncionalista simboliza la victoria de la economía sobre la política y de la razón por sobre la pasión.

Entre las premisas de la teoría de Haas (1958), todavía de gran utilidad y pertinentes para analizar las perspectivas de reunificación de China y Taiwán, están las siguientes. En primer lugar, se considera que los miembros de grupos regionales se perciben más interdependientes a medida que las transacciones formales e informales aumentan. Segundo, mientras menos diferencias perciban en los beneficios y ganancias del proceso de cooperación, evaluarán positivamente seguir colaborando. Tercero, el tamaño de los Estados involucrados no es un indicador decisivo para predecir el éxito o fracaso de la integración que se pueda lograr. Cuarto, el incremento de los canales institucionales en una región, tanto públicos como privados, estimula la interdependencia entre los miembros a medida que estos utilizan los mecanismos de resolución de conflictos establecidos. Y en quinto lugar, la creación del mercado común es la manera más rápida de conducir la integración regional y maximizar el desbordamiento de la cooperación e integración en otras áreas.

La hipótesis que deriva de lo anterior es que a mayor integración económica menor posibilidad de escalar hacia un conflicto militar, porque el costo para ambas partes sería mayor a los costos de la cooperación. Pese a ello, el escenario de las relaciones sino-taiwanesas presenta algunos nudos que, mirados desde el realismo de las relaciones internacionales, permiten analizar las dificultades que enfrenta un eventual proceso de mayor integración entre las partes en condición de iguales. Estos nudos son tres y que ya analizamos en el acápite anterior. Primero, los 1000 misiles que China mantiene apuntando hacia Taiwán como advertencia ante la posibilidad de una declaración formal de independencia (CHINA AUMENTARÍA, 2010); segundo, la alianza entre Taiwán y EE. UU. para equilibrar las fuerzas de ataque de China, y aquí destaca como principal factor la venta de armas a la isla por parte de EE. UU.; y, tercero, la Ley Antisecesión firmada por China el 2005. 


\section{Conclusiones: ¿independencia o avance hacia una reunificación pacífica?}

Según Stephen Krasner, el concepto de soberanía puede entenderse de cuatro formas diferentes, saliendo de las limitantes que establece lo indivisible del concepto: legal internacional, westfaliana, interna e interdependiente. El planteamiento de Krasner resulta interesante para analizar el caso de Taiwán, que funciona como un Estado de facto pero sin reconocimiento internacional. Además, el autor explica que los diferentes tipos de soberanías no tienen por qué irse modificando necesariamente de la misma forma, por lo tanto, un Estado puede tener algunos tipos y no todos. Es precisamente el caso de Taiwán, que no tiene soberanía legal internacional pero sí tiene soberanía interna, westfaliana e interdependiente. Decimos que funciona como un Estado de facto porque tiene autoridad y control sobre su territorio y población, a su vez que mantiene fuera la intervención de terceros actores extranjeros, tiene un sistema democrático con un alto grado de gobernabilidad, y tiene control sobre el flujo a través de sus fronteras. Por otra parte, al no tener soberanía legal internacional, Taiwán no puede ser miembro de organizaciones internacionales pero sí ha firmado acuerdos comerciales con los países con que tiene relaciones diplomáticas formales, como puede ser el caso de El Salvador, Honduras y Guatemala, por mencionar algunos.

$\mathrm{Al}$ respecto, y a modo de conclusiones, podemos preguntarnos si el Acuerdo Marco de Cooperación Económica firmado entre Taiwán y China en 2010 significa avanzar un paso en el reconocimiento de la soberanía legal internacional taiwanesa o bien avanzar hacia una reunificación pacífica de ambas partes.

Desde que la República de China se traslada a la isla esta tiene y ejerce soberanía westfaliana y soberanía interna e interdependiente bajo un gobierno autoritario hasta 1986, comenzando desde entonces un proceso de transición a la democracia. Esta transición, que se inició con la creación de un partido político alternativo, el PPD, como primer partido de oposición, será clave para consolidar las soberanías mencionadas, ahora en un contexto democrático que les da legitimidad en sus expresiones de autoridad y control. En consecuencia, el partido que había gobernado la isla desde 1949, el KMT, vio amenazada su posición de poder y decidió permitir ciertas medidas liberalizadoras que aflojaran su relación autoritaria con la sociedad (RODRÍGUEZ, 2010, 23). Ese primer paso fue el fin de la Ley Marcial ese mismo año, en 1986, permitiéndose la posibilidad de que se formaran otros partidos políticos y que participaran activamente en el debate y la competencia política.

Es importante señalar que la transición de Taiwán no fue desde un régimen militar, sino que fue una transición política pacífica impulsada desde arriba por un gobierno de un solo partido. Dicho partido no perdió su buena reputación ante la sociedad gracias a la estabilidad económica y social que mantuvo, así como por su intento constante de legitimarse como Estado y obtener el reconocimiento de la comunidad internacional. En tal sentido, el inicio de la transición política de Taiwán no fue condicionado por algún tipo de crisis o inestabilidad económica, 
que pudiera haber incentivado las demandas populares hacia algún tipo de reforma política o que fomentara el crecimiento de una oposición al régimen (RODRÍGUEZ, 2010, 200).

Asimismo, para la soberanía interna es necesario considerar que el gobierno del KMT y su control sobre los distintos poderes del Estado, así como sobre los medios de comunicación, estaba institucionalizado. El Estado se había separado del Partido, como en la Europa del Este poscomunista, y los militares y el aparato de seguridad del Estado fueron despolitizados (CHU, 1998, 133). Otro factor que favoreció una transición pacífica hacia la democracia fue la incorporación, en la estructura de poder del KMT, de personas nativas de Taiwán que apoyaron las reformas políticas. Estos nativos taiwaneses participaron de las ideas políticas del KMT y fueron incorporándose en la jerarquía del partido. Eso provocó que en el momento de la transición democrática ellos fueran los principales partidarios del cambio, ya que representaban la realidad de Taiwán por encima de los ideales del gobierno nacionalista que se trasladó desde el continente (CHU, 1998, 134). A su vez, durante la segunda mitad de la década de los ochenta, Taiwán comenzó una etapa de profundización del proceso de democratización, manteniendo un alto crecimiento económico y estableciendo nuevos partidos políticos luego del fin de la Ley Marcial. Así, se realizó una completa reelección del Yuan Legislativo y se logró una reforma constitucional que redefinió el rol de las instituciones y de los actores políticos, estableciéndose un sistema semipresidencial de gobierno en 1997. 3

Este proceso de democratización generó una identidad taiwanesa que se refleja en la construcción y participación de la sociedad en el sistema político democrático. Dicha transferencia de poder a un gobierno representativo de la sociedad conforma la legitimidad interna del Estado taiwanés, que reclama autonomía y reconocimiento internacional. Esto hace difícil que la población taiwanesa acepte ser parte de la R.P. China porque las nuevas generaciones han optado por un gobierno representativo de la sociedad que conforman. En cualquier caso, con las reformas constitucionales que han perfeccionado el funcionamiento democrático del sistema político y tras las segundas elecciones legislativas en 1998 y con la alternancia en el poder en las elecciones del 2000, 2004 y 2008, puede darse por concluido lo esencial de la consolidación democrática.

Sin embargo, es necesario también mencionar que hay dos factores intervinientes importantes e implícitos en el desarrollo político de Taiwán: la presión de China continental por la reunificación con Taiwán, y su inestable posición en el sistema internacional por la falta de un reconocimiento internacional como Estado.

Estos aspectos nos llevan al debate sobre la legitimidad del Estado, que, según el razonamiento de Hung-Mao Tien y Yun-Han Chu (1996, 1140), el

3 El Presidente Lee Teng Hui, a finales del año 1996 organizó la Conferencia de Desarrollo Nacional, con representantes de todos los partidos políticos, de acuerdo a una representación proporcional basada en la fuerza relativa de cada partido. Entre los temas que aquí se resolvieron se encontraba el cambio de la estructura del gobierno central, acordando pasar de un sistema parlamentario estipulado en la constitución a un sistema semipresidencial. 
problema de la legitimidad del Estado es el principal obstáculo para la completa consolidación democrática de Taiwán. No obstante, y retomado el planteamiento de Krasner sobre lo divisible que es el concepto de soberanía, el problema de la legitimidad del Estado para Taiwán tendría una mayor complejidad, es decir, el problema de la legitimidad sería solo parcial, concretamente en lo que atañe a la soberanía legal internacional, pero no así para las otras tres soberanías, que, como hemos analizado, han estado presentes y expresadas en altos niveles de autoridad y control tanto en el periodo de gobierno autoritario como en el momento de la transición y sucesivos gobiernos democráticos.

En este sentido, Taiwán sí ha aceptado la cooperación y las instituciones creadas para retomar contacto con China como parte de su soberanía interdependiente. No obstante, el gobierno taiwanés se entrampa en limitaciones internas y externas. Por un lado, no puede optar por la reunificación con China porque la población taiwanesa mayormente no lo quiere, pero por otro lado, tampoco puede declarar la independencia porque China atacaría la isla, posibilidad que se hace constitucional con la Ley Antisecesión de 2005.

De parte de Taiwán, hay factores que favorecen el statu quo, y que están situados principalmente en las dinámicas de desarrollo político de Taiwán en relación a su proceso de democratización. Hemos identificado dos: en primer lugar, el KMT siempre ha mantenido una posición de rechazo hacia declarar la independencia, y el PPD, partido de oposición en el periodo del gobierno autoritario del KMT (1949-1996) y del gobierno democrático del KMT (19962000), siempre ha usado la declaración de independencia como su bandera de lucha en la competencia política. Sin embargo, al momento de llegar al gobierno, en el 2000, y luego ser reelegido el presidente Chen Shui-bian en el 2004, no hubo declaración de independencia.

Las razones de esto pueden ser simplemente que la campaña presidencial da para decir mucho y luego al llegar al gobierno eso se modera en función de la práctica, hasta razones más empíricas que tienen que ver con el funcionamiento de las instituciones de gobierno taiwanesas, esto es, que Cheng Shui-bian, por ejemplo, no tuvo el apoyo político del Yuan Legislativo para declarar la independencia de la isla. En este sentido, Chen Shui-bian no apoya el principio de una China, para él Taiwán es un Estado soberano e independiente, lo que en la práctica se traduce en no renunciar a declarar formalmente la independencia.

Sin embargo, esta tensión porque gobernantes del PPD declararan la independencia se ha visto desechada tras las últimas elecciones presidenciales y legislativas en Taiwán tras el triunfo del KMT en 2008, generándose de inmediato un acercamiento con la China continental. Lo anterior es relevante en cuanto la afinidad entre China y el KMT está demostrada desde 2005 con una coincidencia de ideas de cooperación más que de confrontación. Este antecedente queda demostrado en los primeros meses del gobierno de Ma, periodo en el cual normalizó las relaciones marítimas, aéreas y postales con el continente. Asimismo 
se reanudaron las relaciones entre la organización Fundación para los Intercambios en el Estrecho y su contraparte china, la Asociación para las Relaciones a Través del Estrecho. Al respecto, durante 2008 y 2009 se han firmado 12 acuerdos entre ambas organizaciones (RÍOS, 2003, 3).

A lo señalado, se suma lo que Xulio Ríos $(2003,4)$ denomina la «tregua diplomática» que tácitamente han acordado ambos gobiernos, demostrada en hechos como: la no oposición de China al ingreso de Taiwán como miembro observador a la Asamblea General de la Organización Mundial de la Salud (OMS); a su vez, países que aun tienen relaciones diplomáticas con Taiwán las han mantenido sin tener una mayor contraofensiva por parte de China y estableciendo relaciones de intercambio de otro tipo con esta última; por último, esta tregua diplomática también se deja ver ante el nuevo anuncio de venta de armamento de EE. UU. a Taiwán realizado en enero de 2010, ante el cual China ha dirigido sus críticas ante el primero, sin mencionar ni una sola vez a Taiwán. La venta sería por un valor de 6400 millones de dólares estadounidenses, incluyendo 114 proyectiles Patriot, 60 helicópteros Black Hawk y equipo de comunicaciones para la flotilla de aviones de combate taiwaneses (CHINA FUSTIGA VENTAS, 2010).

Sin embargo, esa no agresión política que se deja ver entre China y Taiwán desde que gobierna la isla el KMT tiene su mejor expresión en el afianzamiento de los lazos económicos, aspecto posibilitado por el consenso bilateral de la necesidad de intensificar relaciones comerciales. En datos, «el volumen del intercambio comercial en los primeros cuatro meses de 2010 aumentó en un 67,9\%, llegando a los 44260 millones de dólares» (RÍOS, 2003, 2). Esto en un contexto de crisis económica con un alto impacto en la economía taiwanesa que en los años anteriores significó una considerable reducción del crecimiento del PIB y un aumento del desempleo. Desde este punto de vista, las estrechas relaciones económicas entre China y Taiwán en el marco de un gobierno más afín a los intereses del continente han sido los factores fundamentales que han permitido la firma del acuerdo económico en junio de 2010, siendo un paso importante en la construcción de soberanía legal internacional.

En definitiva, hemos analizado el problema de la división de Taiwán de la R.P. China desde 1949 hasta el presente, considerando los factores que favorecen el statu quo del conflicto y aquellos que pueden favorecer un acercamiento y diálogo entre las partes, sin que por ello sostengamos que la solución al problema sea únicamente la reunificación pacífica, sino que también queda abierta la opción de que Taiwán continúe con su construcción de soberanía legal internacional. Asimismo, se produce una convergencia de intereses políticos y económicos contradictorios a ratos, que pueden analizarse desde el realismo y el liberalismo, que, por un lado, favorecen el mantenimiento del statu quo del conflicto, como fue analizado con la Ley Antisecesión, y por otro, favorecen el acercamiento y la cooperación bilateral, como fue analizado con el acuerdo económico. 


\section{Bibliografía}

BAILEY, P.J.: China en el siglo XX. Barcelona: Editorial Ariel, 2002.

CHU, Y.: Taiwan's Unique Challenges. En DIAMOND, L y PLATTNER, M. (Eds.) Democracy in East Asia. Baltimore: The Johns Hopkins University Press, 1998.

CHINA fustiga venta de armas a Taiwán, 30 ene. 2010. En: <http://www.bbc.co.uk/mundo/ internacional/2010/01/100129_0155_taiwan_armas_lf.shtml> [Consulta: 19 ago. 2010].

CHINA aumentaría la cantidad de misiles que apuntan a Taiwán, 21 jul. 2010. Organización Editorial Mexicana. En <http://www.oem.com.mx/laprensa/notas/n1716250.htm> [Consulta: 11 jul. 2010].

DECLARACIÓN del Ministerio de Relaciones Exteriores de China, 17 mar. 2005. Radio Internacional de China, en <http://español.chinabroadcast.cn> [Consulta: 5 feb. 2009].

GALEN CARPENTER T. y DORN James A. (Eds.): China's Future. Washington: Cato Institute, 2000.

HAAS, E.: The Uniting of Europe. Political, Social and Economic Forces, 1950-1957, Stanford: Stanford University Press, 1958.

HALBEISEN, H. (2003): La Política Nacional de Taiwán desde las elecciones presidenciales 2000. Documentos de trabajo sobre Estudios de Asia Oriental, n. ${ }^{\circ} 53$, p. 11-12. En <http://www. unidue.de/-hy0382/fileadmin/publications/gruen/paper53.pdf> [Consulta: 15 mar. 2010].

HUNG M. y CHU Y.: Building Democracy in Taiwán. The China Quarterly, n. ${ }^{\circ}$ 148. Diciembre de 1996.

JACKSON, R. y SORENSEN G.: Introduction to International Relations. Theories and Approaches. Oxford University Press, 2003.

KEOHANE, R. y NYE, J.: Poder e Interdependencia. Buenos Aires: GEL, 1993.

KRASNER, S.: Soberanía, hipocresía organizada. Barcelona: Editorial Paidós, 2001.

LEE, L.: The Unification of China: China-Taiwan relations in flux. Boulder: Lynne Rienner, 1995.

MITRANY, D.: A Working Peace System: An Argument for the Functional Development of International Organization. London: Royal Institute of International Affairs, 1943.

OCDE mejora proyecciones de países BRIC y prevé fuerte expansión para el 2010 y 2011.7 jun. 2010. En <http://www.thefoodlinks.com/web/es/news/ocde-mejora-proyecciones-paisesbric-y-preve-fuerte-expansion-para-2010-y-2011> [Consulta: 15 ago. 2010].

OFICINAS COMERCIALES.ES (2010): Coyuntura político-económica de Taiwán. En <http://www.oficinascomerciales.es/icex/cda/controller/pageOfecomesGen/0,5936,5398971_ 5399866_5503715_0_TAI,00.html> [Consulta: 14 ago. 2010].

POCH DE FELIU, R. (2005): China aprueba su ley antisecesión. Marzo. En <http://www. lainsignia.org> [Consulta: 10 dic. 2009].

PRESUPUESTO defensa de China en el 2010 aumentará en un 7,5 por ciento. 4 mar. 2010. En <http://lta.reuters.com/article/topNews/idLTASIE62306O20100304> [Consulta: 23 ago. 2010].

REINOSO, José (2010): China y Taiwán firman el acuerdo comercial más importante de su historia. 29 jun. 2010. En <http://www.elpais.com/articulo/internacional/China/Taiwan/ 
firman/acuerdo/comercial/importante/historia/elpepuint/20100629elpepuint_6/Tes> [Consulta: 3 ago. 2010].

REPUBLICA POPULAR CHINA (2000): Libro Blanco: El Principio de una sola China y el problema de Taiwán. Beijing: 21 de febrero. En <http://www.taiwandocuments.org/white.htm> [Consulta: 22 ago. 2010].

RIOS, X. (2010): Taiwán: el primer bienio de Ma Ying-jeou. Revista ARI del Real Instituto Elcano. No 93. En <http://www.realinstitutoelcano.org>.

RIGGER, Séller: Politics in Taiwan. Ed. London: Routledge, 1999.

RODRIGUEZ I.: Continuidad politica y cambio económico en la China del siglo XXI. Santiago de Chile: RIL Editores, 2010

TOW, W.T.: Asia-Pacific Strategic Relations: Seeking Convergent Security. Cambridge University Press, 2001.

WALTZ, K.: Man, the State and War, a theoretical analysis. Nueva York: Columbia University Press, 1959.

WU, J.: On Deng Xiaoping Thought. Beijing: Foreign Languages Press, 1996

Recibido en 13 de septiembre de 2010 Aceptado el 31 de marzo de 2011

\section{Resumen}

El presente artículo tiene por objetivo analizar el problema de la reunificación entre la República Popular China y Taiwán en la última década, en el marco de dos hitos: por un lado, la promulgación de la Ley Antisecesión del 14 de marzo de 2005 y, por otro, la firma del Acuerdo Marco de Cooperación Económica el 30 de junio de 2010.

Palabras clave: China; Taiwán; reunificación; realismo; liberalismo.

\section{Abstract}

This article aims to analyze the problem of reunification between the PRC and Taiwan in the last decade, as part of two milestones: first, the enactment of the Anti-Secession Law of March 14, 2005; and second, the signing of the Economic Cooperation Framework Agreement on 30 June 2010.

Keywords: China; Taiwan; Reunification; Realism; Liberalism. 